\title{
Frequency Behavior of an MMC Test Bench System
}

\author{
Matthias Quester, Fisnik Loku, Viswaja Yellisetti, Albert Moser \\ Institute for High Voltage Equipment and Grids, Digitalization and Energy Economics, RWTH Aachen University \\ Aachen, Germany \\ m.quester@iaew.rwth-aachen.de
}

\begin{abstract}
Interactions of modular multi-level converters (MMCs) compromising the system stability can be assessed by investigating the frequency behavior of the converter. The MMC Test Bench system replicates full-scale converters on a low-voltage level. It can emulate high-voltage direct current systems representing meshed offshore grids as well as the influence of wind farms connected to MMCs. Measuring the MMC Test Bench's frequency behavior allows for assessing the frequency behavior of the applied control systems by avoiding simplifications of the electrical part of the MMC. This paper presents a setup for measuring the frequency-dependent impedance of the laboratory MMCs. The impedance is compared to small-scale as well as fullscale converter models. It is shown that the measured impedance can be scaled up for full-scale HVDC studies by means of derived correction factors. The results further demonstrate that simplified time-domain models can represent the MMC Test Bench but fail to represent the exact frequency behavior at low and high frequencies.
\end{abstract}

Index Terms-Impedance Measurement, MMC Test Bench, Impedance Scaling, Harmonic Stability

\section{INTRODUCTION}

Modern power systems consist of alternate current (AC) as well as high-voltage direct current (HVDC) systems to transmit power over long distances and to connect load centers with generation sources [1]. HVDC systems often are coupled with the AC system by means of voltage source converters, more specifically modular multi-level converters (MMCs), which offer high controllability and low harmonic distortion. MMCs can nonetheless interact with the AC grid impedance, compromising the system stability if resonances are excited by the MMC's control system. To assess this risk, the frequency behavior of the MMC can be modeled as frequencydependent impedance and evaluated in combination with the grid impedance for stability studies. The MMC impedance can be derived analytically by linearizing the MMC's control system in the frequency domain. While this approach offers insight into factors influencing the frequency behavior, it cannot model every physical phenomena influencing the frequency behavior. Additionally, the complexity of the control system prevents including every existing control loop. A method to overcome these challenges can be to model the MMC's impedance by measuring the frequency response of a physical converter test bench. However, test benches have been used for validation of analytical models only [2], [3]. They operate on lower power and voltage ratings compared

The authors' work has received funding from the European Union's Horizon 2020 research and innovation program under grant agreement No 691714. to HVDC converters, and the effect of scaling down the ratings is neglected. This paper investigates the influence of scaling down on the frequency behavior of an MMC. The frequency-dependent impedance of an MMC Test Bench (TB) at RWTH Aachen University is measured and compared to the impedance of a simulated, down-scaled small-scale (SS) test bench model to investigate first differences resulting from comparing simulations and physical components. The impact of scaling down the ratings is subsequently investigated by comparing the impedance of the MMC TB with that of a fullscale (FS) model obtained from an HVDC system used for grid studies. The same control system is applied to the MMC TB, the SS model and the FS model so that differences due to the electrical system can be investigated.

\section{MMC TEST BenCH System}

As shown in Fig. 1, the MMC TB consists of eight laboratory-scaled MMCs, thirty-two П-line sections and four linear TBs (PAs). The MMC TB can be separated into a DC grid and an AC grid.

\section{A. DC Grid}

The DC grid of the MMC TB consists of lab-scaled MMC stations. Each converter of the MMC TB is based on MOSFETs and consists of six arms comprising an arm inductor and ten submodules connected in series. One realtime simulator (RTS), an OPAL-RT OP4510, serves as a DC grid controller to provide reference operating points for each

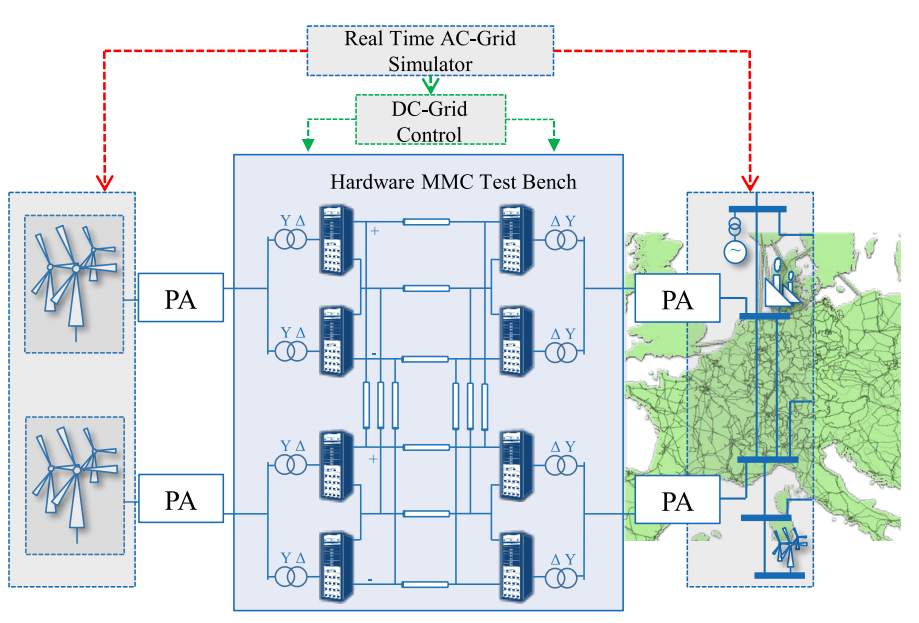

Fig. 1. Schematic design of the MMC TB [4] 
TABLE I

CONVERTER PARAMETERS.

\begin{tabular}{|l|c|}
\hline Converter power & $6 \mathrm{~kW}$ \\
\hline DC voltage & $400 \mathrm{~V}$ \\
\hline Arm indcutance $L_{a r m}$ & $2.5 \mathrm{mH} \pm 15 \%$ \\
\hline No. of submodules $n_{\text {sub }}$ & 10 \\
\hline Submodule capacity & $4.92 \mathrm{mF} \pm 15 \%$ \\
\hline Nominal Cell Voltage & $40 \mathrm{~V}$ \\
\hline MOSFET Switching Frequency & $0-10 \mathrm{kHz}$ \\
\hline Transformer rated power & $10 \mathrm{kVA}$ \\
\hline Transformer configuration & $Y / \Delta$ \\
\hline Transformer $U_{\text {Primary }}$ & $400 \mathrm{~V}$ \\
\hline Transformer $U_{\text {Secondary }}$ & $208 \mathrm{~V}$ \\
\hline
\end{tabular}

MMC station if required. Furthermore, each MMC contains one RTS (OP4510) that serves as converter controller with the upper level controls implemented on the Central Processing Unit (CPU). The lower level control, i.e. the sub-module sorting and the modulation, is implemented on the Field Programmable Gate Array (FPGA) of the RTS. The MMC ratings are presented in TABLE I] [4].

Cascaded $\Pi$-line sections with lumped capacitors and inductors represent the DC line connections between the MMCs. Lengths of $1600 \mathrm{~km}$ and $800 \mathrm{~km}$ can be emulated for monopolar and bipolar network configuration, respectively.

\section{B. AC Grid}

The AC grid is represented by a Thevenin equivalent with a voltage source and an equivalent grid impedance . AC grids are implemented on RTS using the electromagnetic transient (EMT) simulation software Hypersim or the Matlab toolbox Simulink in combination with RT-Lab for real-time simulation. The linear PAs with a bandwith of $25 \mathrm{kHz}$ at $-3 \mathrm{~dB}$ allow for the coupling between the hardware DC grid and the simulated AC systems. The RTS outputs the signals as voltages between $-10 \mathrm{~V}$ and $10 \mathrm{~V}$. The signals are sent to the PA through the analogue outputs and control the PA which can operate either as voltage or current source.

\section{MMC Control}

Fig. 2 shows the MMC control system using a cascaded vector control scheme [5].

The dispatch controller provides the reference signals, i.e. the active power, reactive power, DC voltage and AC voltage reference. The upper-level control regulates the corresponding measured signals to reach the provided reference values and consequently, by controlling the AC current, it provides the reference arm voltages to the lower level control. The lower

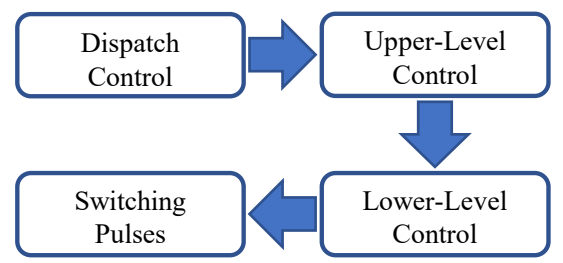

Fig. 2. Schematic design of the MMC control. level control modulates the reference arm voltages and the sorts the switching signals. Lastly, the switching signals are provided to the MMC arms. In the MMC control, the reference signals are provided in per-unit (p.u). The measured signals are accordingly converted to p.u. before they are provided to the controller. The p.u. conversion is performed using the base values of the $\mathrm{AC}$ voltage and current, $\mathrm{DC}$ voltage and current as well as of the submodule voltage and current. The general description of the p.u. conversion is provided in (1).

$$
x_{(\text {p.u. })}=X_{\text {measured }} / X_{\text {base }}
$$

The per-unitization of the control parameters offers the possibility for the MMC control system to be used for different systems regardless of their voltage level. Thus, the simulated systems at high voltage levels and the corresponding SS models can be compared directly. Furthermore, a comparison between the simulated models and a demonstration using the MMC TB can be conducted.

\section{MMC Impedance Measurement Setup}

The MMC TB system shown in Fig. 3 is modified for measuring the impedance of laboratory MMCs. The impedance measurement setup displayed in Fig. 4 can be divided into two parts, the MMC TB and the real-time simulation. The MMC TB consists of two MMCs connected by $\Pi$-line sections on the DC side. Transformers shift the grid voltage given by the PAs, from $208 \mathrm{~V}$ to $400 \mathrm{~V}$ as required by the MMCs. The grid voltage at the primary side of the transformers is supplied by the PAs amplifying the voltage signals received from the RTS. The real-time simulation is coupled with the MMC TB via analogue input and output ports. The ports transmit the control signals for and the measurement signals from the PAs as voltages between $-10 \mathrm{~V}$ and $10 \mathrm{~V}$. The control signals comprise the three-phase grid voltages set at the PAs. While the voltage signal for PA 2 consists only of the grid voltage $U_{\text {Grid }}$, the voltage signals for PA 1 additionally include the perturbation

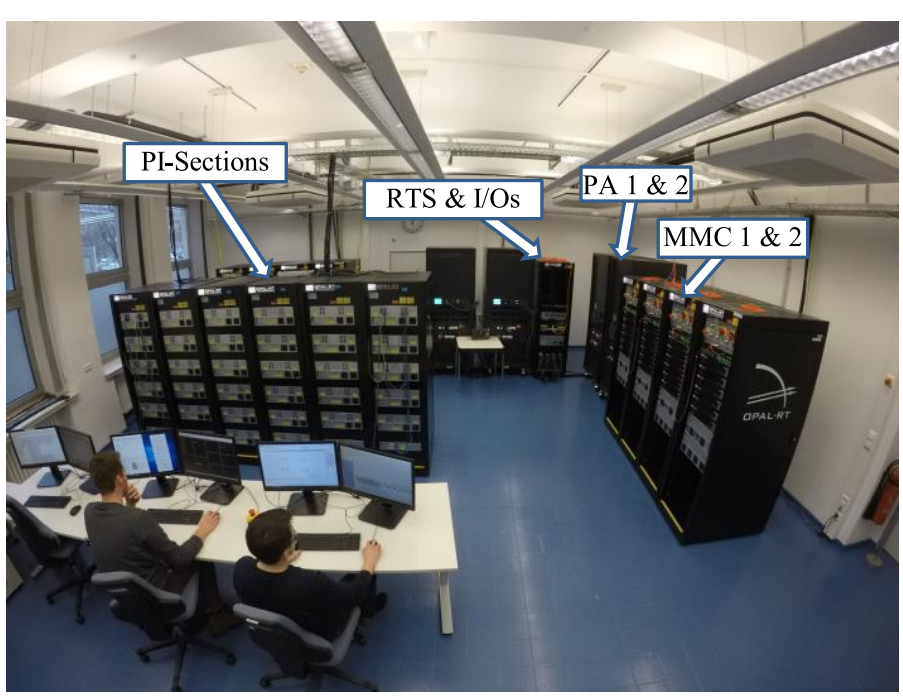

Fig. 3. MMC Test Bench at RWTH Aachen University. 


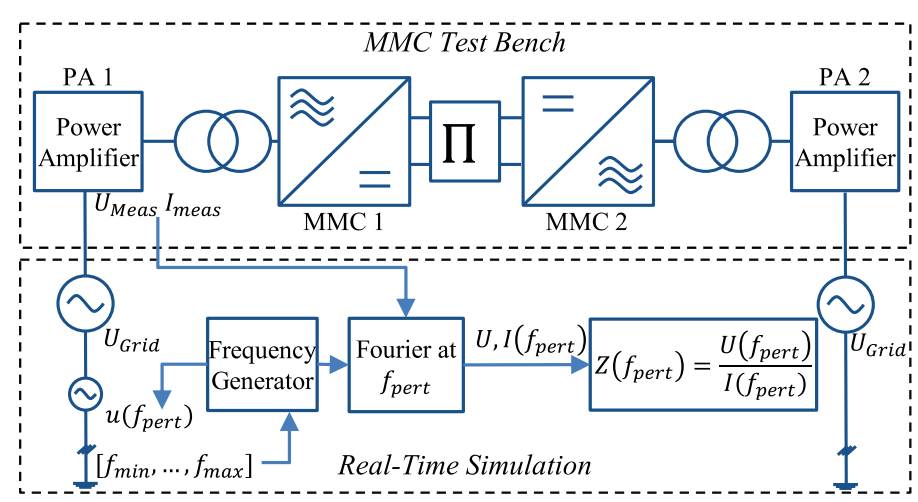

Fig. 4. MMC Test Bench impedance measurement setup.

voltage source $u\left(f_{\text {pert }}\right)$. In case the MMC under investigation works as grid forming converter, PA 1 operates as current source with a $50 \mathrm{~Hz}$ current for setting an operation point in addition to the perturbation current at $f_{\text {pert }}$. The perturbation voltage source is part of the frequency measurement method [6]. Its frequency, $f_{\text {pert }}$, is time-dependent and determined by the frequency generator. The frequency generator provides the frequencies iteratively over time according to the userdefined minimum and maximum frequency $f_{\min }$ and $f_{\max }$ and the desired resolution in addition to the measurement time period. The measured signals consist of the voltage $U_{\text {meas }}$ and the current $I_{\text {meas }}$ measured at the terminal of PA 1. The signals are evaluated in real-time by Fourier analysis dependent on the perturbation frequency, $f_{\text {pert }}$ provided by the frequency generator. The determined voltage $U\left(f_{\text {pert }}\right)$ and current $I\left(f_{\text {pert }}\right)$ at $f_{\text {pert }}$ can then be used to calculate the frequency-dependent impedance $Z\left(f_{\text {pert }}\right)$ of the MMC.

Although only the impedance of MMC 1 is measured, the impedance measurement setup utilizes two MMCs. Thus, the operation mode of the converters can be changed while the system is running. For instance, changing the converters' outer control loops from active power control to DC voltage control does not require a shut down and restart of the overall system. The PAs' control modes can remain in AC voltage source when the outer control mode is changed as opposed to a system with only one converter with one PA on the DC side. Such system would require the PA to act either as a DC voltage source or a DC load dependent on the converter's operation mode. As a result, the impedance measurements can be automatized with different measurement sequences being taken in a continuous run.

\section{MODELING}

\section{A. MMC Simulation Models}

The MMC TB can be simulated offline for preliminary studies and testing of new control algorithms. In addition, a FS HVDC model allows for investigating the impact of scaling so that results obtained with the MMC TB can be used for HVDC studies. The electrical models of the MMC are developed on the basis of an average value model representing the MMC's capacitor voltages as a single voltage source. The MMC TB's
TABLE II

PARAMETERS SIMULATION MODELS.

\begin{tabular}{|r|c|c|}
\hline & SS model & FS model \\
\hline Converter power & $6 \mathrm{~kW}$ & $1200 \mathrm{MW}$ \\
\hline DC voltage & $400 \mathrm{~V}$ & $640 \mathrm{kV}$ \\
\hline Arm indcutance $L_{\text {arm }}$ & $2.5 \mathrm{mH}$ & $46.2 \mathrm{mH}$ \\
\hline Arm resistance $R_{\text {arm }}$ & $0.013 \Omega$ & $0.08 \Omega$ \\
\hline On resistance $R_{\text {on }}$ & $0.925 \mathrm{~m} \Omega$ & $0.08 \Omega$ \\
\hline No. of submodules $n_{\text {sub }}$ & 10 & 350 \\
\hline Submodule capacity & $4.92 \mathrm{mF}$ & $8.8 \mathrm{mF}$ \\
\hline Transformer $U_{\text {Primary }}$ & $400 \mathrm{~V}$ & $400 \mathrm{kV}$ \\
\hline Transformer $R_{\text {Primary }}$ & $0.04 \Omega$ & $0.31621 \Omega$ \\
\hline Transformer $L_{\text {Primary }}$ & $0.219 \mathrm{mH}$ & $0.403 \mathrm{mH}$ \\
\hline Transformer $U_{\text {Secondary }}$ & $208 \mathrm{~V}$ & $350 \mathrm{kV}$ \\
\hline Transformer $R_{\text {Secondary }}$ & $0.032 \Omega$ & $0.726 \Omega$ \\
\hline Transformer $L_{\text {Secondary }}$ & $0.17765 \mathrm{mH}$ & $0.92473 \mathrm{mH}$ \\
\hline Grid strength $S_{\text {SC }}$ & $150 \mathrm{~kW}$ & $300 \mathrm{MW}$ \\
\hline Grid resistance $R_{\text {grid }}$ & $0.1838 \Omega$ & $0.9192 \Omega$ \\
\hline Grid inductance $L_{\text {grid }}$ & $5.8517 \mathrm{mH}$ & $29.2583 \mathrm{mH}$ \\
\hline
\end{tabular}

control system described in section II-C can be applied to both simulated MMC models and the MMC TB, because it is independent of the system's power and voltage ratings due to the per-unitization. The simulated SS and FS models are implemented in Matlab Simulink with the addition of the OPAL-RT block set for real-time simulation. Although realtime simulations have no inherent benefits when not coupled to physical components except potentially faster simulation times, using the real-time time simulators also for simulations only allows for consistent and comparable modeling. For instance, the same impedance measurement method used for measuring the MMC TB's impedance can be used for the simulated models. The simulated models' parameters are given in TABLE[I] The SS model's parameters are chosen according to the MMC TB's specifications to match simulations and laboratory tests. Transformer resistances and inductances are calculated according to the transformer's voltage and power ratings based on literature values as only the transformer ratings are specified. The FS parameters are set to represent an HVDC system.

\section{B. Impedance Scaling}

Using the measured MMC TB impedance for high-voltage system stability studies with FS models requires scaling the impedance according to the high-voltage system's voltage level and power rating. Because the control system is in p.u., differences in the impedance depend on the electrical parameters only. Consequently, the electrical parameters of the MMC TB, and its SS model, as well as the FS model are used to calculate a complex correction factor $\underline{k}$ which, when multiplied, scales the MMC TB $\underline{Z}_{T B}$ and the SS impedance $\underline{Z}_{S S}$ to the higher voltage level and ratings. The correction factor is divided into $|k|$ and $k_{\varphi}$ so that for an impedance $\underline{Z}=|Z| \angle \varphi_{Z},\left|Z_{\text {scaled }}\right|=|Z| \cdot|k|$ and $\varphi_{Z_{\text {scaled }}}=\varphi_{Z}-k_{\varphi}$ as

$$
|k|=\sqrt{\frac{r_{F S}^{2}+\left(\omega \cdot l_{F S}\right)^{2}}{r_{S S, T B}^{2}+\left(\omega \cdot l_{S S, T B}\right)^{2}}}
$$



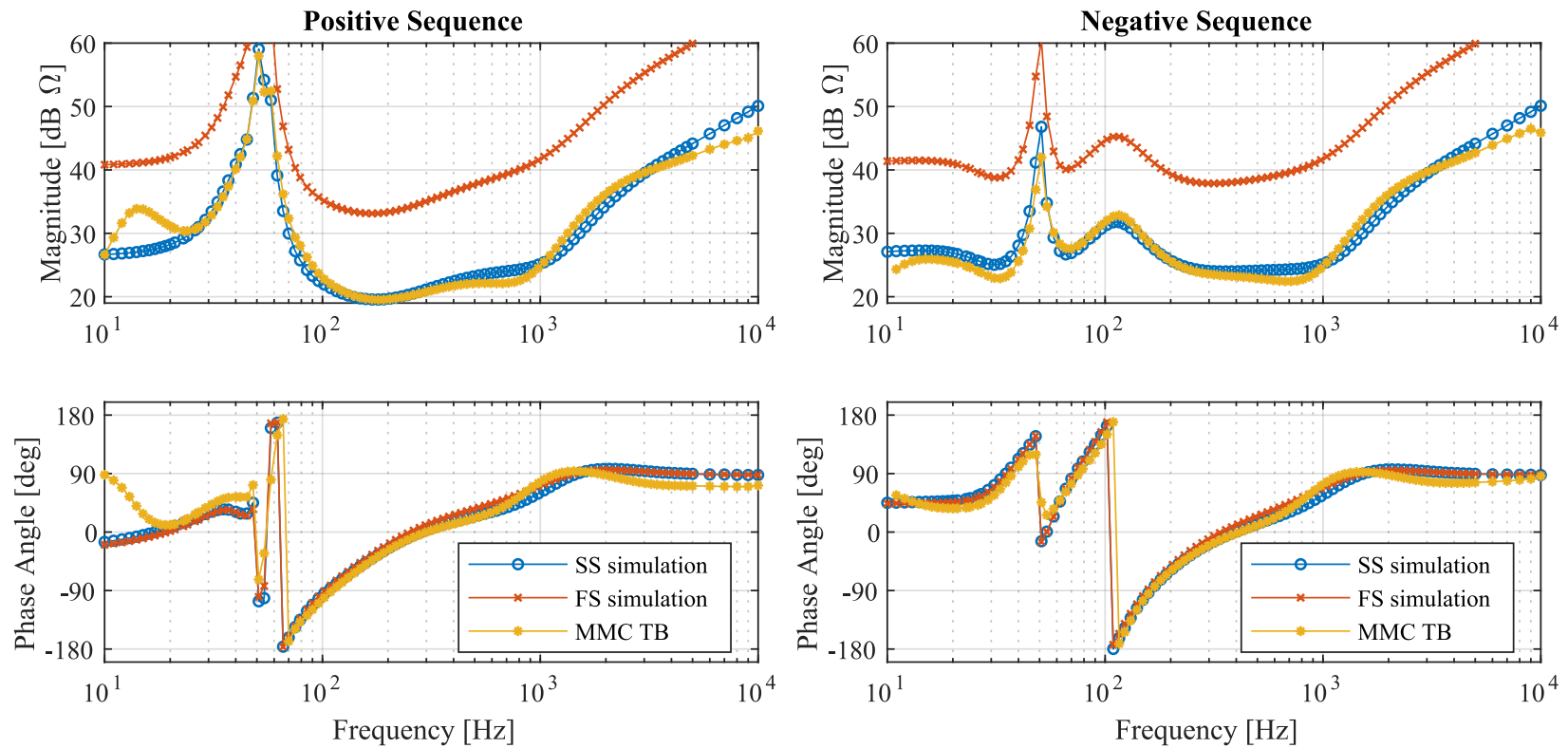

Fig. 5. MMC impedance.

and

$$
k_{\varphi}=\arctan \frac{r_{F S}}{\omega \cdot l_{F S}}-\arctan \frac{r_{S S, T B}}{\omega \cdot l_{S S, T B}} .
$$

The equivalent resistances $r_{S S, T B}$ and $r_{F S}$ are calculated according to the resistive SS model, MMC TB and FS model parameters where

$$
r=R_{\text {grid }}+R_{\text {Primary }}+R_{\text {Secondary }}^{\prime}+R_{\text {arm }}^{\prime}+n_{\text {sub }} \cdot R_{\text {on }}^{\prime}
$$

The equivalent inductances $l_{S S}$ and $l_{F S}$ are calculated according to the inductive SS model, MMC TB and FS model parameters, respectively:

$$
l=L_{\text {grid }}+L_{\text {Primary }}+L_{\text {Secondary }}^{\prime}+L_{\text {arm }}^{\prime} .
$$

Variables denoted with ' are referred from the secondary side of the transformer to the primary side. The SS model and the MMC TB parameters used for calculating $\underline{k}$ are identical because the SS model is modeled according to the MMC TB's specifications. The grid impedance, $R_{\text {grid }}+j \omega L_{\text {grid }}$, is based on the grid's short circuit power $S_{S C}$. The angular frequency, $\omega$, is a vector and equals the product of $2 \pi$ and the perturbation frequency $f_{\text {pert }}$, meaning that the calculated correction factor $\underline{k}$ is a vector as well and needs to be multiplied by $\underline{Z}$ for

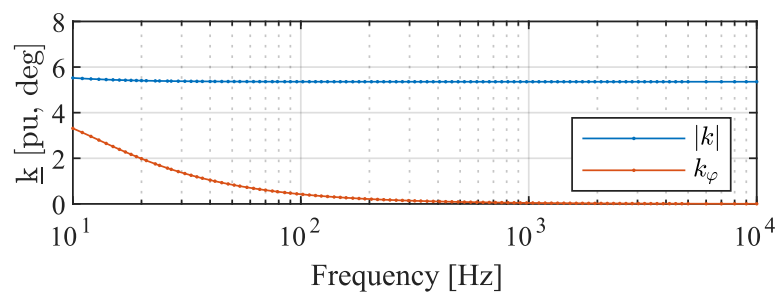

Fig. 6. Correction factors. every frequency. Fig. 6 shows $|k|$ and $\left|k_{\varphi}\right|$ calculated for the MMC TB and SS model according to the parameters given in TABLE II] It can be seen that the magnitude of the FS model is expected to be 6 times larger than that of the MMC TB and the SS model, according to the difference in the electrical parameters. In contrast, only at low frequencies does scaling the MMC impedances affect the phase angle as it can be seen that for high frequencies $k_{\varphi}$ converges to zero degree. For high frequencies, $\omega$ dominates $\underline{k}$ resulting in $|k|$ being dependent on $l_{F S} / l_{S S}$ only and $k_{\varphi}$ not being affected by the electrical parameters.

\section{RESUlts}

The impedance measuring method is applied to the simulated SS and FS models as well as to the MMC TB. Fig. 5 shows the results for positive and negative sequence perturbations. The simulated models and the MMC TB operate as symmetrical monopoles with MMC 1 controlling the active power on the $\mathrm{AC}$ side and transmitting $0.5 \mathrm{pu}$ from $\mathrm{MMC} 1$ to MMC 2, and MMC 2 controlling the DC voltage. Using a series of 100 perturbation frequencies on a logarithmic scale, the measuring method determines the magnitude and phase angle of the impedance of MMC 1 from $10 \mathrm{~Hz}$ to $10 \mathrm{kHz}$.

\section{A. MMC Impedances}

Comparing the impedances of the simulated MMC models and the MMC TB shows that the high-voltage rating of the FS model results in an impedance higher than that of the SS model and the MMC TB, both operating on a lowvoltage level. Contrarily, the phase angle is not affected by the different voltage levels as it mainly depends on the ratio of resistive and inductive parameters being similar for the high-voltage and low-voltage MMCs. Furthermore, Fig. 5 

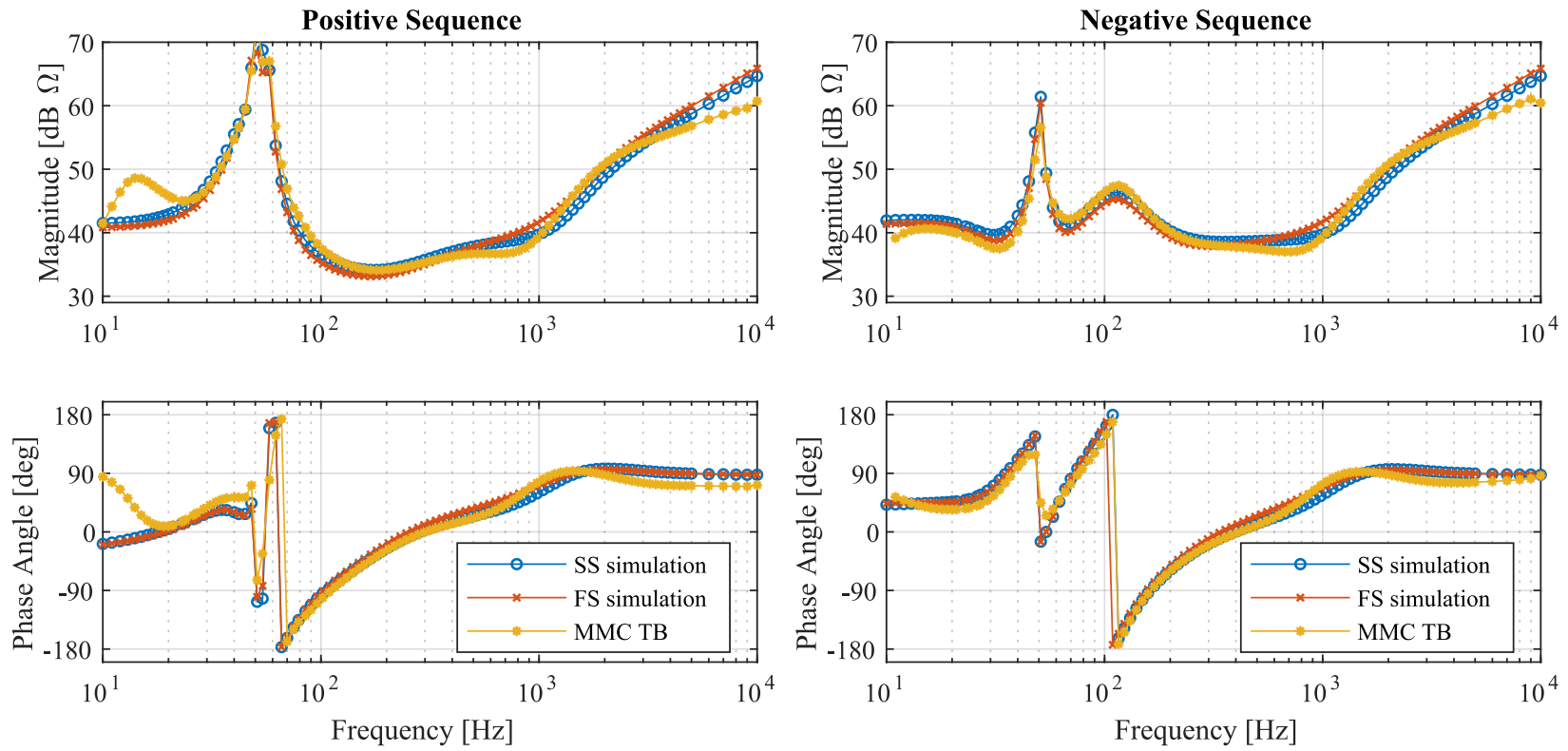

Fig. 7. MMC impedance scaled.

demonstrates that the impedances of the simulated SS model and the MMC TB match except for low frequencies below the fundamental frequency, frequencies between $300 \mathrm{~Hz}$ and $1 \mathrm{kHz}$ and very high frequencies above $2.5 \mathrm{kHz}$. The deviations can be attributed to different effects influencing the MMC impedances. The resonance peak at $15 \mathrm{~Hz}$ in positive sequence indicates that an internal circulating current resonance exists in the MMC TB which the simulated MMC models cannot replicate [7] due to its simplified electrical structure. As it is pointed out in [8], internal dynamics such as capacitor voltage variations can cause series and parallel resonances at low frequencies. At frequencies around $800 \mathrm{~Hz}$, the MMC TB's impedance shows a higher damping in both positive and negative sequence. With increasing frequency, it can be seen that the MMCs show purely inductive behavior. However, above $4 \mathrm{kHz}$, the impedance of the simulated MMC impedances differ significantly. While the simulated models continue to show purely inductive behavior, the magnitude of the MMC TB impedance is increasing less with higher frequencies and the phase angle is $18^{\circ}$ smaller. Above $4 \mathrm{kHz}$ winding stray capacitances significantly impact the frequency response of transformers [9]. This effect is not included in the converter transformer models of the simulation and therefore explains the different frequency behavior at very high frequencies. Furthermore, due to tolerances of the passive components of the MMC TB, the given parameters might not be exactly as specified. In addition, transformer parameters need to be estimated on the basis of given specifications. Comparing positive and negative sequence impedances reveals resonance peaks existing in positive or negative sequence only at $15 \mathrm{~Hz}$ and $120 \mathrm{~Hz}$, respectively.

\section{B. MMC Impedances Scaled}

The correction factors presented in Fig. 6 are applied to the measured impedance of the SS model and the MMC TB. Fig. 7 shows the up-scaled impedances of the SS model and the MMC TB in comparison to the FS model's impedance. It can be seen that the correction factor, $|k|$, leads to closer matching of the MMC TB's impedance magnitude with that of the FS model except for frequency regions where the frequency behavior of the SS model and MMC TB are affected by phenomena not being modeled in the simulations.

\section{CONCLUSION AND OUTLOOK}

A measurement setup was presented for determining the frequency-dependent impedance of the MMC TB. The measurement setup enables investigations of the frequency behavior of physical laboratory MMCs by measuring their impedance without the need for data-heavy post-processing. Thus, various control systems and their impact on the frequency behavior can be quickly assessed because the measurement can be automatized and no additional tedious modeling of the MMC's control system is required in the frequency domain. By using a physical laboratory test bench, frequency phenomena not included in models can be revealed. The results were compared to an SS model, a representation of the MMC TB in Matlab Simulink, and an FS model simulating an HVDC system.

The results show that the magnitude of the FS model's impedance is higher compared to that of the SS model and the MMC TB due to higher impedances of the electrical system. Comparing the impedance of the MMC TB and the SS model demonstrates that the model is able to match the MMC TB's magnitude and phase angle with the exception at 
very high frequencies above $5 \mathrm{kHz}$ and low frequencies where a parallel resonance can be seen in the MMC TB impedance. Consequently, by applying control systems on the MMC Test Bench, frequency phenomena can be revealed and identified which might be neglected in simulations due to simplifications in the models. Around $800 \mathrm{~Hz}$ the physical setup experiences a higher damping resulting in a lower magnitude of the impedance. In contrast, the phase angle does not differ between the FS and SS model. Because the electrical parameters are scaled accordingly, the ratio of the real and imaginary part of the electrical parameters is equal in the SS and FS models. Also, it can be seen that the phase angle is similar for all impedance measurements. Small differences in the phase angle occur at low frequencies only because at high frequencies, the inductive parameters dominate the phase angle as it can be seen in (3). The differences between the SS model and the MMC TB illustrate that assumptions and simplifications when modeling MMCs need to be carefully considered with respect to the range of frequency under investigation.

Evaluating the SS and FS parameters makes it possible to define correction factors which can be used for scaling the low-voltage level MMC TB impedance according to a desired voltage level. The comparison of the up-scaled MMC TB impedance with the FS model's impedance illustrates that the up-scaled impedances can be used for HVDC stability studies as it is proven that the impedance's magnitude is in accordance with an FS-MMC's impedance. Phase angles need to be corrected if a different $X / R$ ratio is assumed for the HVDC MMC and only for frequencies below $100 \mathrm{~Hz}$ when the phase angle is not dominated by the inductive behavior of the MMC.

Scaling up by predefined correction factors according to the difference in electrical parameters allows for using the measured down-scaled MMC TB impedance for system stability studies with FS grids. Because it could be seen that applied models failed to replicate the MMC TB's frequency behavior in particular frequency regions, future work will investigate weather more complex time-domain models are able to narrow down the frequency phenomena observed with the MMC TB. Operating the MMC Test Bench in open loop will clarify which effects can be contributed to the control system and which are caused by the electrical part of the MMC. Also, the effect of frequency coupling on frequencies will be investigated and included in the measured impedance [10]. Future work will also utilize the developed impedancemeasurement test bench and investigate the impact of different MMC control systems such as grid forming control and the impact on stability studies. Further on, measured impedances will be scaled for FS grid studies including offshore wind farms.

\section{REFERENCES}

[1] ENTSO-E, "Ten-year network development plan 2016 - executive report," pp. 42-61, 2016.

[2] L. Bessegato, L. Harnefors, K. Ilves, and S. Norrga, "A method for the calculation of the ac-side admittance of a modular multilevel converter,"
IEEE Transactions on Power Electronics, vol. 34, no. 5, pp. 4161-4172, May 2019.

[3] J. Sun and H. Liu, "Sequence impedance modeling of modular multilevel converters," IEEE Journal of Emerging and Selected Topics in Power Electronics, vol. 5, no. 4, pp. 1427-1443, Dec 2017.

[4] P. Ruffing, Y. Sun, Y. Yang, I. Jahn, S. Norrga, Ömer Gösku, N. Cutululis, M. Kaiser, M. Quester, F. Loku, C. Petino, R. Blasco-Gimenez, S. Bernal-Perez, and L. Zeni, "Deliverable 16.1: Definition and specification of test cases," 2018. [Online]. Available: https://www.promotion-offshore.net/fileadmin/PDFs/D16.1_ Definition_and_Specification_of_Test_Cases.pdf

[5] C. W. B4.57, "Guide for the development of models for hvdc converters in a hvdc grid," CIGRÉ technical brochure 604, 2014.

[6] M. Quester, F. Loku, V. Yellisetti, and R. Puffer, "Online impedance measurement of a modular multilevel converter," in 2019 IEEE PES Innovative Smart Grid Technologies Europe (ISGT-Europe), Sep. 2019, pp. $1-5$.

[7] J. Lyu, X. Zhang, X. Cai, and M. Molinas, "Harmonic state-space based small-signal impedance modeling of a modular multilevel converter with consideration of internal harmonic dynamics," IEEE Transactions on Power Electronics, vol. 34, no. 3, pp. 2134-2148, March 2019.

[8] H. Wu, X. Wang, . Kocewiak, and L. Harnefors, "Ac impedance modeling of modular multilevel converters and two-level voltage-source converters: Similarities and differences," in 2018 IEEE 19th Workshop on Control and Modeling for Power Electronics (COMPEL), June 2018, pp. $1-8$.

[9] A. Bonner, T. Grebe, E. Gunther, L. Hopkins, M. Marz, J. Mahseredjian, N. Miller, T. Ortmeyer, V. Rajagopalan, S. Ranade, P. Ribeiro, B. Shperling, T. Sims, and W. Xu, "Modeling and simulation of the propagation of harmonics in electric power networks. i. concepts, models, and simulation techniques," IEEE Transactions on Power Delivery, vol. 11, no. 1, pp. 452-465, Jan 1996.

[10] I. Vieto and J. Sun, "Sequence impedance modeling and convertergrid resonance analysis considering dc bus dynamics and mirrored harmonics," in 2018 IEEE 19th Workshop on Control and Modeling for Power Electronics (COMPEL), June 2018, pp. 1-8. 